P.M. Putora*, G. Benz" ${ }^{\#}$ R. Rodriguez ${ }^{\mp}$, M. Brutsche ${ }^{\#}$ and M. Früh ${ }^{+}$

*Dept of Radiation Oncology, "Dept of Pneumology, "Institute of Pathology, and ${ }^{+}$Dept of Oncology and Hematology, Cantonal Hospital St Gallen, St Gallen, Switzerland.

Correspondence: P.M. Putora, Klinik für Radio-Onkologie, Kantonsspital St Gallen, 9007 St Gallen, Switzerland. E-mail: paul.putora@kssg.ch

Statement of Interest: None declared.

\section{REFERENCES}

1 Segaert S, Van Cutsem E. Clinical signs, pathophysiology and management of skin toxicity during therapy with epidermal growth factor receptor inhibitors. Ann Oncol 2005; 16: 1425-1433.

2 Tang X, Shigematsu H, Bekele BN, et al. EGFR tyrosine kinase domain mutations are detected in histologically normal respiratory epithelium in lung cancer patients. Cancer Res 2005; 65: 7568-7572.
3 Bolognia JL, Jorizzo JL, Rapini RP, eds. Dermatology. St Louis, Mosby, 2007.

4 Weiss SW, Goldblum JR, Enzinger FM, eds. Enzinger and Weiss's Soft Tissue Tumours. 5 Edn. St Louis, Mosby, 2008.

5 Lee MW, Seo CW, Kim SW, et al. Cutaneous side effects in nonsmall cell lung cancer patients treated with Iressa (ZD1839), an inhibitor of epidermal growth factor. Acta Derm Venereol 2004; 84: 23-26.

6 Boucher KW, Davidson K, Mirkhur B, et al. Paronychia induced by cetuximab, an antiepidermal growth factor receptor antibody. J Am Acad Dermatol 2002; 45: 632-633.

7 Dainichi T, Tanaka M, Tsuruta N, et al. Development of multiple paronychia and periungual granulation in patients treated with gefitinib, an inhibitor of epidermal growth factor receptor. Dermatology 2003; 207: 324-325.

8 Nakano J, Nakamura M. Paronychia induced by gefitinib, an epidermal growth factor receptor tyrosine kinase inhibitor. J Dermatol 2003; 30: 261-262.

9 Chang GC, Yang T-Y, Chen K-C, et al. Paronychia and skin hyperpigmentation induced by gefitinib in advanced non-small-cell lung cancer. J Clin Oncol 2004; 22: 4646-4648.

DOI: $10.1183 / 09031936.00008411$

\title{
Atypical pulmonary carcinoid tumour in a 28-year-old nonsmoker with Prader-Willi syndrome
}

\section{To the Editors:}

In recent years, the life expectancy of patients with Prader-Willi syndrome (PWS) has increased, unveiling several cases of earlyonset cancer and raising the question whether the condition predisposes to tumourigenesis. We encountered a unique occurrence of pulmonary atypical carcinoid in a young neversmoker with PWS.

A 28-yr-old, morbidly obese male with PWS was admitted as a candidate for gastric bypass. He was a lifetime nonsmoker, and his height, weight and body mass index were $1.78 \mathrm{~m}, 175 \mathrm{~kg}$, and $55.2 \mathrm{~kg} \cdot \mathrm{m}^{-2}$, respectively. Polyphagia, obesity and a failure to achieve psychosocial milestones became apparent at the age of 5 yrs, when PWS was diagnosed based on clinical findings and a de novo deletion of the proximal region of chromosome 15 (del(15)(q11-q13)). Type II diabetes mellitus was diagnosed at the age of $17 \mathrm{yrs}$, and dietary control along with administration of sulfonylurea was initiated. The remainder of the anamnesis included low levels of testosterone, which were treated with monthly intramuscular depot injections of testosterone enanthate $(250 \mathrm{mg})$.

On admission, the patient reported mild shortness of breath, nonproductive cough and malaise of indeterminate origin. A physical exam revealed markedly increased subcutaneous fat, picking scars, hypoplastic external genitalia and a polyphonic wheeze over the right hemithorax. Pulse was regular and of normal rate, diastolic blood pressure was elevated $(98 \mathrm{mmHg})$ and haemoglobin oxygen saturation was $96 \%$ in room air. Electrocardiography was normal. Laboratory data, including blood cell counts, routine serum biochemistry and coagulation times, were within normal limits, except for fasting plasma glucose $\left(178 \mathrm{mg} \cdot \mathrm{dL}^{-1}\right)$ and lipids (total cholesterol $233 \mathrm{mg} \cdot \mathrm{dL}^{-1}$; high-density lipoprotein cholesterol $32 \mathrm{mg} \cdot \mathrm{dL}^{-1}$; triglycerides $\left.322 \mathrm{mg} \cdot \mathrm{dL}^{-1}\right)$.

A pre-operative chest radiograph, performed routinely but also prompted by the patient's wheeze, showed a round, regularshaped opacity of soft-tissue quality measuring $7 \times 5 \mathrm{~cm}$ superimposed on the right pulmonary hilum (fig. 1a). Computed tomography confirmed a $7.5 \times 6.5 \times 5 \mathrm{~cm}$ well-demarcated mass of soft-tissue density partially attached to the posteromedial structures of the right hemithorax in very close proximity to the posterior mediastinum (fig. 1b). Fibreoptic bronchoscopy revealed a pale tumour with well-defined margins measuring $\sim 1 \mathrm{~cm}$ in diameter and obliterating the bronchus intermedius by $90 \%$ (fig. 1c). The endobronchial portion of the tumour rested on a relatively limited base and was bronchoscopically removed.

Histopathological examination of endobronchial biopsies (fig. 1d-i) showed an atypical carcinoid with focal areas of necrosis (fig. 1d), two to 10 mitoses per $2 \mathrm{~mm}^{2}$ detected by Ki67 immunostaining (fig. 1e), high cytokeratin 8 expression indicating epithelial origin (fig. 1f), and high CD56, chromogranin and synaptophysin expression indicating neuroendocrine differentiation (fig. 1g-i) [1]. There was no evidence of neoplasms of the pituitary, pancreas and parathyroid, as determined by brain magnetic resonance imaging and octreotide scanning. The tumour was pre-operatively staged as T3N0M0 (stage IIB) according to the 2009 TNM7 system, as the primary tumour measured $>7 \mathrm{~cm}$, but no locoregional or distant metastases were found [2]. Surgical resection was decided upon, and middle and lower bilobectomy was 

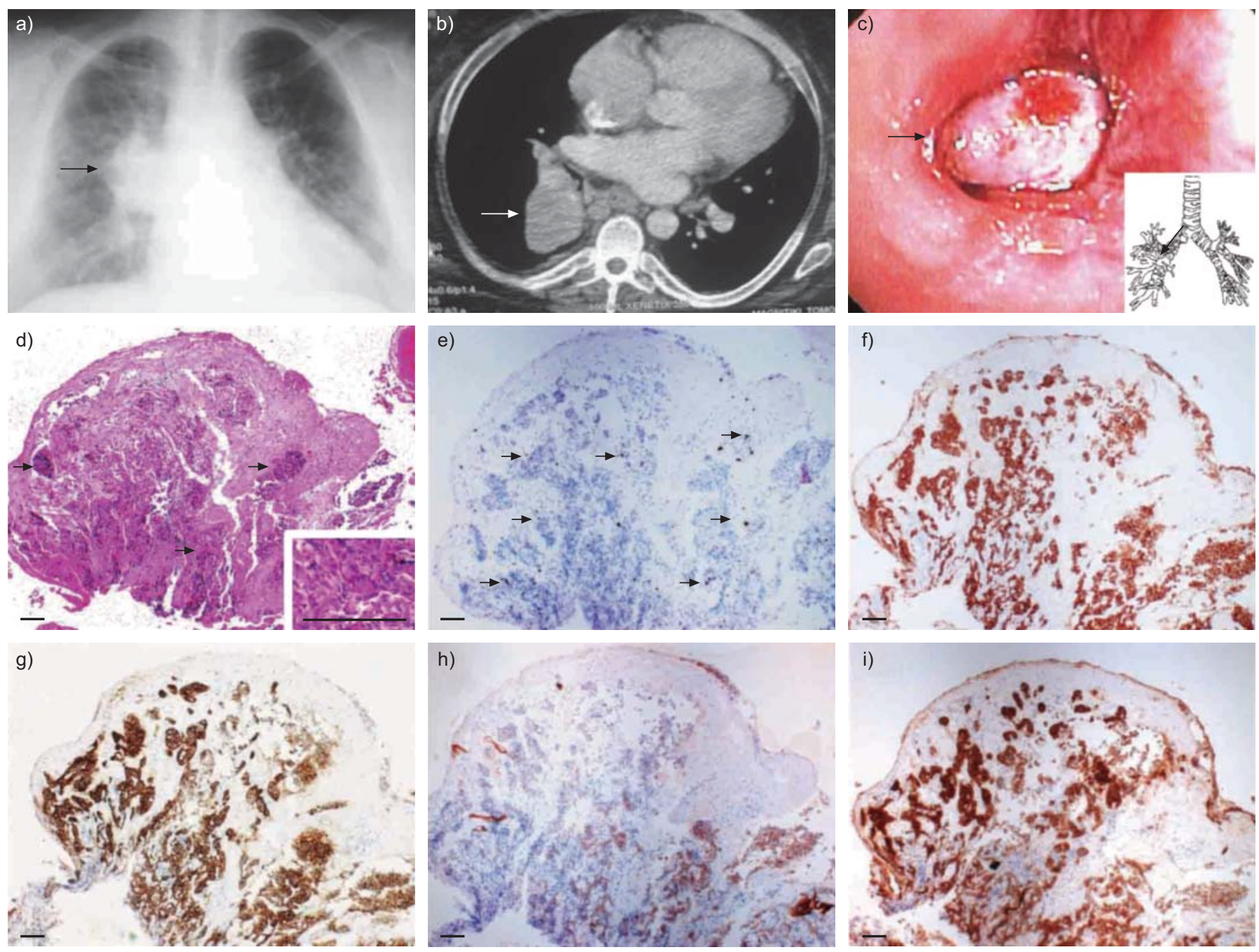



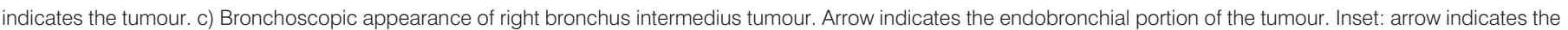

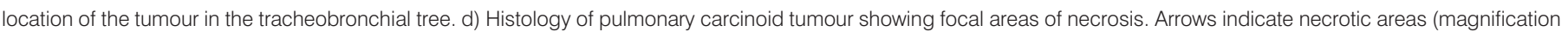

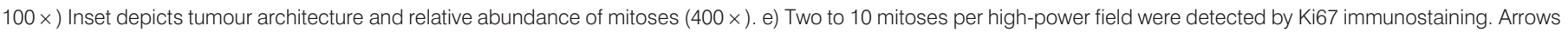

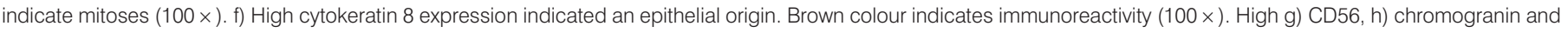
i) synaptophysin expression indicated neuroendocrine differentiation. Brown colour indicates immunoreactivity $(100 \times)$. Scale bars $=100 \mu \mathrm{m}$.

successfully performed, including removal of mediastinal lymph nodes. Post-operative histopathology showed a radical resection of all tumour with clear operative margins and no infiltration of lymph nodes by carcinoid tissue. The intra- and post-operative course was uneventful and the tumour had not recurred after 2 yrs of follow-up.

PWS, a multisystemic neurogenetic disorder first reported in 1956, affects $\sim 1$ in 25,000 individuals. Commonly associated clinical features include infantile central nonprogressive hypotonia, hyperphagia, obesity, short stature, dysmorphism, small hands and feet, distinctive facial features, mental retardation, hypogonadism, hypogenitalism and cryptorchidism [3]. PWS is correlated to significant morbidity and mortality, with an associated death rate of $3 \%$ per year. However, with improved care, the life expectancy of patients with PWS has increased considerably in recent years, unmasking possible protumourigenic susceptibility [4]. Several reported cases of malignancy in persons with PWS, including Wilms' tumour, leukaemia, lymphoma, seminoma, ovary teratoma, hepatoblastoma and multiple endocrine neoplasia type I, have raised the question of whether PWS predisposes to cancer, similar to the increased cancer risk associated with other congenital disorders with chromosomal defects [5]. To our knowledge, this is the first report of an atypical pulmonary carcinoid in a patient with PWS.

PWS is due to a combination of genomic imprinting (i.e. allelespecific silencing of gene expression via promoter methylation) combined with genetic lesions or alterations in Mendelian patterns of chromosomal inheritance and is regarded as a prototype of inherited epigenetic disorders [3]. In this regard, a critical region at bands q11-13 on maternal chromosome 15 is silenced by methylation; only the paternal allele is expressed. Hence, if this expressed paternal allele is lost via deletion, translocation, imprinting defect or inheritance of both chromosomes 15 from the mother, this region is not expressed at all. 
Approximately $70 \%$ of patients with PWS present a deletion of the paternal chromosome 15 at band q11-13 (del(15)(q11-q13)), whereas the syndrome develops in $25-30 \%$ as a result of maternal uniparental disomy for chromosome 15 ; in $<1 \%$ of cases, the molecular abnormality is an imprinting defect [3].

Atypical carcinoids, tumours of intermediate malignancy in the spectrum of pulmonary neuroendocrine tumours, are rare: they represent $20 \%$ of pulmonary carcinoids, which, in turn, account for only $1 \%$ of all lung tumours in the USA, with an age-adjusted incidence rate of 0.5 per 100,000 population [6]. In contrast to typical carcinoid, atypical pulmonary carcinoid tumours present at an older mean age (65 versus 55 yrs) and are possibly epidemiologically associated with smoking [1]. Pulmonary carcinoids may arise solitarily or may contribute to the spectrum of multiple endocrine neoplasia type 1 syndrome. Atypical pulmonary carcinoid is diagnosed histologically, based on the presence of necrosis (which is never observed with typical carcinoid) and an intermediate mitotic index of two to 10 mitoses per $2 \mathrm{~mm}^{2}$ (10 high-power fields of view on most microscopes); typical carcinoids, i.e. the low-grade counterparts of atypical carcinoids, have less than two mitoses per $2 \mathrm{~mm}^{-2}$, whereas high-grade small cell and large cell neuroendocrine lung cancers feature $>10$. Genetic abnormalities documented in atypical carcinoids include $3 p$ deletions in $73 \%$ of cases, loss of heterozygosity of the retinoblastoma gene in $22 \%$ and of $11 \mathrm{q} 13$ in 50\%, and TRP53 abnormalities in 45\% [1, 7].

Based on the present and previous reports of early-onset malignancies in patients with PWS, it is intriguing to hypothesise that one or more important tumour suppressor genes are located within the critical del(15)(q11-q13) region imprinted and/or deleted in PWS. Indeed, Necdin (NDN) is such a candidate tumour suppressor located within this chromosomal region. NDN protein functions similarly to the retinoblastoma (RB)1 tumour suppressor protein and interacts with tumourrelated protein (TRP)53 to facilitate TRP53 degradation [8]. Interestingly, although NDN was identified as a gene imprinted in human and mouse, reduced expression of which might be responsible for PWS, mouse models of $N d n$ loss did not link $N d n$ with PWS [9]. Instead, evidence suggests that $N d n$, as well as other genes located on $\operatorname{del}(15)(\mathrm{q} 11-\mathrm{q} 13)$, such as MKRN3, MAGEL2 and SNURF-SNRPN, may function as tumour suppressors in various tissues [8]. Indeed, cross-examining the genetic lesions found in pulmonary carcinoid tumours and PWS, one can hypothesise a possible pathway of carcinoid development in patients with PWS. In this regard, if a stabilising mutation occurs in the TRP53 tumour suppressor gene on an inherited background of PWS-associated NDN gene loss, excess nonlabile (i.e. mutant) TRP53 protein may accumulate intracellularly, leading to prevention of apoptosis of tumour-initiated neuroendocrine cells and carcinoid formation. In support of this hypothesis, approximately one-fifth of pulmonary carcinoids that develop in patients without inherited genetic disorders lack both or one of the two functional RB1 alleles, as well as normal TRP53 [1]. Since NDN functions complimentary to the RB1 tumour suppressor, it is plausible to hypothesise that, in patients with PWS who, by definition, lack functional NDN, TRP53 mutations may be more sustainable by initiated cells, leading to lung carcinoid tumour formation. In this regard, combined $R b 1$ and Trp53 inactivation in the respiratory epithelium has been shown to trigger high-grade neuroendocrine lung neoplasia in mice [10]. In humans with PWS, combined NDN and TRP53 inactivation could similarly present critical events. leading to pulmonary carcinoid tumours or their more aggressive counterparts, small and large cell neuroendocrine lung cancers.

In conclusion, we present a unique case of atypical bronchial carcinoid in a young patient with PWS. Together with published observations of early-onset neoplasia in patients with PWS, the case reported herein suggests a possible link between genetic lesions present in PWS and enhanced/early onset carcinogenesis in several organ systems. In the lungs, inherited loss of NDN, a gene contained within the critical locus on chromosome 15 that is deleted and/or imprinted in PWS, together with mutations in TRP53 and/or RB1, genes known to be frequently mutated in pulmonary neuroendocrine tumours, could provide a mechanistic link between PWS and enhanced neoplasia. As the survival of PWS patients gradually increases, this relationship is worthy of investigation.


A. Kokkori ${ }^{\S}$, P. Dedeilias*, J. Kokotsakis*, M. Argiriou* and C. Zisis*

*Depts of Cardiothoracic Surgery, ${ }^{\#}$ Radiology, ${ }^{+}$Critical Care and Pulmonary Services, and ${ }^{\S}$ Histopathology, General Hospital "Evangelismos", Athens, and "Dept of Physiology, Faculty of Medicine, University of Patras, Rio, Greece.

Correspondence: G.T. Stathopoulos, Dept of Physiology, Faculty of Medicine, University of Patras, Basic Biomedical Sciences Research Building, 2nd floor, Room B40, 1 Asklepiou Str., University Campus (Panepistimioupolis), 26504 Rio, Greece. E-mail: gstathop@upatras.gr

Statement of Interest: None declared.

\section{REFERENCES}

1 Bertino EM, Confer PD, Colonna JE, et al. Pulmonary neuroendocrine/carcinoid tumors: a review article. Cancer 2009; 115: 4434-4441.

2 Groome PA, Bolejack V, Crowley JJ, et al. The IASLC Lung Cancer Staging Project: validation of the proposals for revision of the T, $\mathrm{N}$, and $\mathrm{M}$ descriptors and consequent stage groupings in the forthcoming (seventh) edition of the TNM classification of malignant tumours. J Thorac Oncol 2007; 2: 694-705.

3 Buiting K. Prader-Willi syndrome and Angelman syndrome. Am J Med Genet Part C Semin Med Genet 2010; 154C: 365-376.

4 Whittington JE, Holland AJ, Webb T, et al. Population prevalence and estimated birth incidence and mortality rate for people with Prader-Willi syndrome in one UK health region. J Med Genet 2001; 38: 792-798.

5 Rainier S, Feinberg AP. Genomic imprinting, DNA methylation, and cancer. J Natl Cancer Inst 1994; 86: 753-759.

6 Travis WD, Lubin J, Ries L, et al. United States lung carcinoma incidence trends: declining for most histologic types among males, increasing among females. Cancer 1996; 77: 2464-2470.

7 Voortman J, Lee JH, Killian JK, et al. Array comparative genomic hybridization-based characterization of genetic alterations in pulmonary neuroendocrine tumors. Proc Natl Acad Sci USA 2010; 107: 13040-13045. 
8 Chapman EJ, Knowles MA. Necdin: a multi-functional protein with potential tumor suppressor role? Mol Carcinog 2009; 48: 975-981.

9 Tsai TF, Armstrong D, Beaud AL. Necdin-deficient mice do not show lethality or the obesity and infertility of Prader-Willi syndrome. Nat Genet 1999; 22: 15-16.
10 Meuwissen R, Linn SC, Linnoila RI, et al. Induction of small cell lung cancer by somatic inactivation of both Trp53 and Rb1 in a conditional mouse model. Cancer Cell 2003; 4: 181-189.

\section{Clinical features of central airway involvement in autoimmune pancreatitis}

\section{To the Editors:}

Autoimmune pancreatitis (AIP) is characterised by a high serum immunoglobulin (Ig)G4 concentration and various extrapancreatic complications, including those of the lung [1]. Therefore, AIP is currently not viewed as a separate disease entity, but as the pancreatic involvement of a systemic IgG4related disease [2]. We previously reported a patient with AIP showing central airway stenosis and bilateral hilar lymphadenopathy (BHL) similar to sarcoidosis [3]. Following this case, we prospectively identified an additional five patients with AIP who revealed similar airway findings (and BHL) in our department from September 2007 to January 2009. The six patients (including the first patient) met the diagnostic criteria of AIP proposed by Japanese Pancreatic Society in 2006 [1]. In order to update the available clinical and therapeutic information on central airway involvement in AIP, we performed airway biopsies and other examinations in these six patients.

All six patients gave their written informed consent for the performance of bronchoscopy and blood sampling. The angiotensin-converting enzyme (ACE) and IgG4 concentrations were measured at Special Reference Laboratories Inc. (Tokyo, Japan), and interleukin (IL)-6 was measured at Mitsubishi Kagaku Bio-Clinical Laboratories Inc. (Tokyo, Japan). Chest computed tomography (CT) was performed with a multidetector row helical CT scanner (LightSpeed VCT; GE Medical Systems, Milwaukee, WI, USA) with both inspiratory and expiratory views [4]. All CT images were reviewed by two radiologists (S. Kawakami and Y. Fujinaga, Shinshu University School of Medicine, Matsumoto, Japan) [4]. Fibreoptic bronchoscopy (BF 1T-240 or 1T-260; Olympus corporation, Tokyo, Japan) was performed, and bronchoalveolar lavage (BAL), transbronchial lung biopsy (TBLB) and bronchial biopsy were achieved according to our routine protocol [5]. The TBLB was carried out in the right upper lobe (S2 and S3) and the bronchial biopsy was performed at the right second spur and the upper lobe bronchi. Immunohistochemical staining was performed by affinity-purified sheep polyclonal antibody against human IgG4 (The Binding Site Group Limited, Birmingham, UK). The numbers of both IgG4- and IgG-positive cells were counted in regions of the highest cellular density and averaged for three high-power fields (HPFs) by one pathologist (T. Uehara) [6]. Wilcoxon signed-rank test was used to compare the measurements before and after treatment.
Patient profiles, laboratory data, chest CT findings, pulmonary function tests (PFTs) and bronchoscopic data are shown in table 1. Four out of our six patients with airway involvement associated with AIP were middle-aged men, similar to the epidemiological features described in our previous study on patients with AIP [1]. Cough without dyspnoea or fever was present in all six patients. Although serological tests did not show particular abnormalities in terms of lactate dehydrogenase (LDH), C-reactive protein (CRP), ACE and IL-6, the serum levels of IgG and IgG4 were extremely high in all patients (table 1). BHL was observed in all six patients. We have previously reported hilar lymphadenopathy to be present in $\sim 78 \%$ of the patients with AIP, as detected by chest CT [4]. The most characteristic finding on chest $\mathrm{CT}$ imaging was thickening of the bronchial wall and bronchovascular bundles (fig. 1a). The PFTs (table 1) showed no restrictive ventilatory defects; however, airflow limitation was observed in several patients (forced expiratory volume in $1 \mathrm{~s}$ (FEV1)/forced vital capacity (FVC) $<70 \%$ in four and $70-80 \%$ in two patients; median $69.7 \%$, range $60.5-77.6 \%$ ) and the median FEV1 was $2.87 \mathrm{~L}$ (range 1.56$3.60 \mathrm{~L}$ ). The bronchoscopic examinations showed irregular stenoses of the bronchus and bronchi accompanied by mucosal oedema and engorged vessels (fig. $1 \mathrm{~b}$ and c) in all patients. In the BAL fluid (BALF), there were high lymphocyte counts with CD4 predominance in all patients, increased eosinophil counts in four patients and low plasma cell counts in all patients. In three out of four patients, the eosinophil count was only slightly elevated. The bronchial biopsy specimens of all patients revealed diffuse airway inflammatory infiltrates, consisting mainly of plasma cells and lymphocytes, together with fibrosis. Although there were scattered eosinophils in the bronchial tissues of all patients, typical findings of asthma (i.e. goblet cell hyperplasia and subepithelial fibrosis) [7] were not observed in any of the bronchial biopsy specimens. Because airway hypersensitivity was not even observed in case 4 , who showed the highest number of eosinophils in BALF, we thought that these findings were a characteristic feature of airway involvement in AIP other than typical asthma. Moreover, these pathological findings of the airway were similar to the other extrapancreatic lesions in AIP [1]. The immunohistochemical examinations showed massive infiltrations of many IgG4positive plasma cells in the bronchial biopsy specimens in all patients. The TBLB specimens of three out of the six patients demonstrated many IgG4-positive plasma cells in the peripheral bronchus and bronchiole; however, few IgG4-positive plasma 